\title{
Negative Refraction Slabs with Varying Indexed Metamaterial
}

\author{
Yao Shen* \\ School of Criminal Science and Technology, People's Public Security University of China, Beijing 100038, China \\ *Corresponding author: shenyaophysics@hotmail.com
}

\begin{abstract}
Negative refraction is a specific optical phenomenon made possible by a negative index material. A slab with negative refraction is an alternative to a normal lens. This type of lens is significantly superior to an ordinary lens. Negative refraction lenses with a single index -1 had been discussed by Pendry in recent decades. Our research focuses on an analytical and numerical method to demonstrate the optical properties and restrictions of negative refraction slabs with a gradient index. The position of focus changes depending on the incident angle. Different from normal varying indexed slab, we give the reason why the negative refraction slabs with varying index could not make a perfect lens. Only negative refraction slab with single index -1 could focus lights on one point, thus make a perfect lens.
\end{abstract}

Keywords: negative refraction, perfect lens, optics

Cite This Article: Yao Shen, "Negative Refraction Slabs with Varying Indexed Metamaterial." International Journal of Physics, vol. 6, no. 2 (2018): 33-37. doi: 10.12691/ijp-6-2-2.

\section{Introduction}

A negative index material (NIM) is a type of material where both permittivity and permeability are negative simultaneously. This phenomenon is called negative refraction. NIMs were first introduced by Veselago in 1968 [1]. In the following years, Pendry et al. [2,3,4,5] gave details about negative refraction and suggested that realizing negative refraction was feasible with molecules with non-trivial broken symmetry, which were called split-ring resonators (SRRs) respectively. Two years later, an experiment was conducted by Shelby et al. [6]. In recent decades, NIMs have become popular in terms of optical properties in electromagnetic fields. For instance, a perfect lens can be produced with a NIM, where the image quality and sensitivity are significantly improved compared with those of an ordinary lens [7,8,9]. Recently, extended metal atom chains (EMACs) with SRRs, anapole moment of different broken symmetry configuration, invisible cloaking, etc. [10-30] have been reported. Pendry gave negative refraction lens with a single index -1 as an example and pointed that it was a perfect lens. Varying indexed slab of normal refraction could act as a normal lens. In this letter, we give the theoretical derivation and calculation of a negative refraction slab with a gradient index to research whether it could make a perfect lens too.

This paper is organized as follows: In section 2, we analyze the theoretical results of a negative refraction slab with a gradient index. The numerical results and simulations are discussed in section 3 . In section 4 , we discuss the influence of interference. The conclusion and anticipation of future work are discussed in section 5 .

\section{Theoretical Derivation}

\subsection{Slabs with Two Negative Indices}

In this part, we discuss a negative refraction slab with two different negative indices (see Figure 1). The distance between the optical source and slab is assumed to be $L$. The thickness of the slab is $D=d_{1}+d_{2}$, where $d_{1}$ and $d_{2}$ are the thicknesses of two different negative index slabs that compose the whole slab. The two different negative indices are $n_{1}<0$ and $n_{2}<0$, where $\left|n_{1}\right|>\left|n_{2}\right|$. The medium outside the material is considered to be air, thus, $n_{0}=1$. The length between the second focus and the material is f. Angles $\theta, \alpha, \beta, \gamma$ are shown in Figure 1. According to Snell's law, we have

$$
\left\{\begin{array}{l}
\sin \theta=n_{1} \sin \alpha, \\
n_{2} \sin \varphi=n_{1} \sin \alpha, \\
n_{2} \sin \varphi=\sin \gamma .
\end{array}\right.
$$

Therefore, we can conclude that the incident angle is equal to the refraction angle, $\theta=\gamma$. Additionally, geometrical relationships are given by

$$
\left\{\begin{array}{l}
f \tan \gamma=\left(d_{2}-x\right) \tan \varphi, \\
x \tan \varphi+d_{1} \tan \alpha=L \tan \theta .
\end{array}\right.
$$

Pendry pointed out that a negative refraction lens could focus light twice [2]. The position of the second focus is 


$$
f=\frac{d_{1} \cos \theta}{\sqrt{n_{1}^{2}-\sin ^{2} \theta}}+\frac{d_{2} \cos \theta}{\sqrt{n_{2}^{2}-\sin ^{2} \theta}}-L .
$$

When light moves vertically towards the material, $\theta=0^{\circ}$, $f=\frac{d_{1}}{n_{1}}+\frac{d_{2}}{n_{2}}-L$.

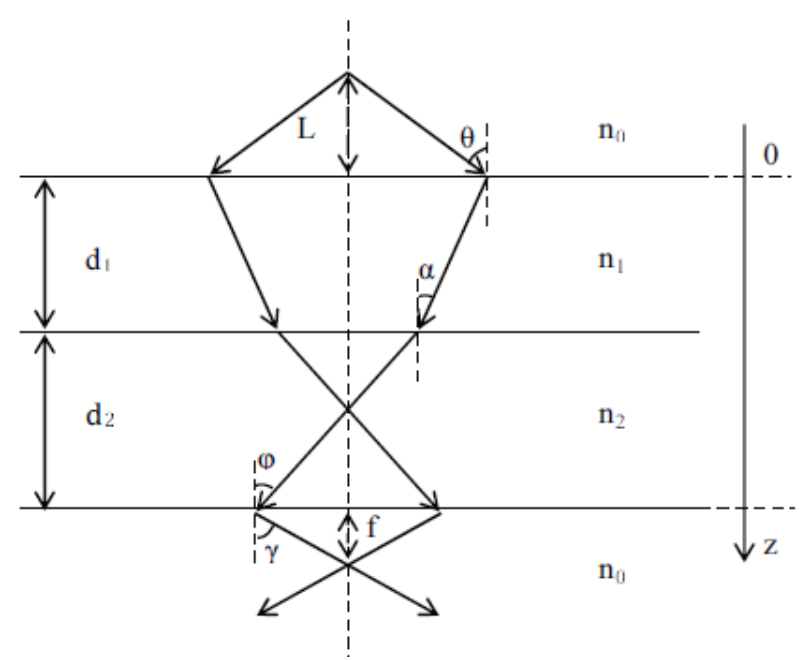

Figure 1. Negative refraction slab with two different negative indices

\subsection{Slab with Linear Negative Index}

According to Pendry's theory [2], because light could transmit through negative refraction slab with no reflection, negative refraction slab with a gradient index can be seen as several layers with constant index. When the negative index of a slab is linear, for instance,

$$
n(z)=-1+a z, z \in[0, D],
$$

where $a$ is a constant, according to (3), we have

$$
\begin{aligned}
& f=\int_{0}^{D} \frac{\cos \theta d z}{\sqrt{(-1+a z)^{2}-\sin ^{2} \theta}}-L \\
& =\frac{\cos \theta}{a} \ln \frac{\left|1-a D+\sqrt{(-1+a z)^{2}-\sin ^{2} \theta}\right|}{1+\cos \theta}-L
\end{aligned}
$$

Because we assume $n(z)<0$, we have $a<\frac{1}{z} \Rightarrow a<\frac{1}{D}$. The discussion of a negative index can be divided into two cases,

- $n(z) \leq-1$

$$
n(z) \in(-\infty,-1] \Rightarrow-\infty<a \leq 0 .
$$

- $-1<n(z)<0$

$$
n(z) \in(-1,0) \Rightarrow 0<a<\frac{1}{D} .
$$

In the first case, the incident angle could be $0^{\circ} \leq 0<90^{\circ}$. When $\theta=0^{\circ}$, the focus reads $f=\frac{d_{1}}{n_{1}}+\frac{d_{2}}{n_{2}}-L$. The angle $\theta$ cannot be $90^{\circ}$ because we only consider the situation in which light moves toward the material. In this case, $f<0$ is reasonable (see Figure 2). Here, light is divergent outside the slab and is only focused once inside.

In the second case, the total internal reection (TIR) should be considered. Because $|n|<1(-1<n(z)<0)$, the incident angle should be $0 \leq \theta<i_{c}$, where $i_{c}$ is the angle of TIR. When $\theta=i_{c}, \quad \alpha=90^{\circ}$. Next, we set $D=2$ $(0 \leq z \leq 2)$, as an example. In accordance with (5) and (7), we have $0<a<\frac{1}{2}$. Here, we assume the focus $f>0$ (see Figure 3) and $0<L<D$. Using these assumptions, we have $\theta=30^{\circ} \Rightarrow a<\frac{1}{11}, \quad \theta=45^{\circ} \Rightarrow a<\frac{1}{15}, \quad$ and $\theta=60^{\circ} \Rightarrow a<\frac{1}{26}$. Calculation tells us that the constant a decreases with increasing incident angle $\theta$. In other words, large $\theta$ gives more restrictive $a$. Because $0 \leq \theta<i_{c}$, the range of a should be small and be decided by $i_{c}$.

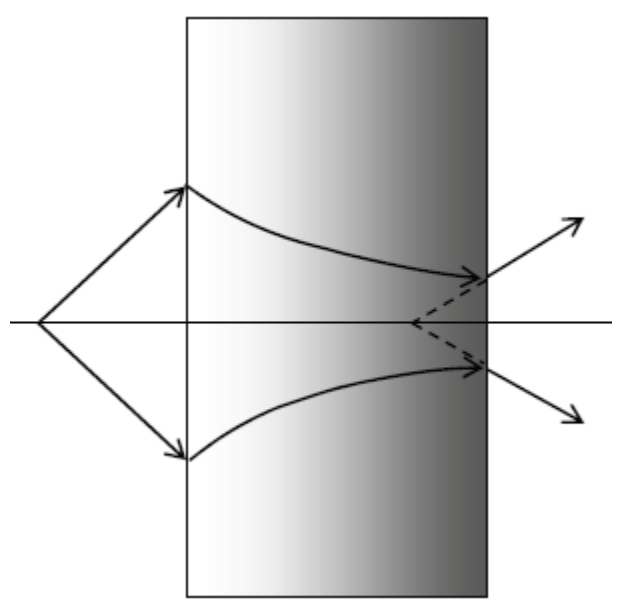

Figure 2. The diagrammatic sketch of a slab with linear negative indices in the first case. The color concentration of the slab represents the absolute value of the negative index. The deeper the color is, the greater the absolute value is

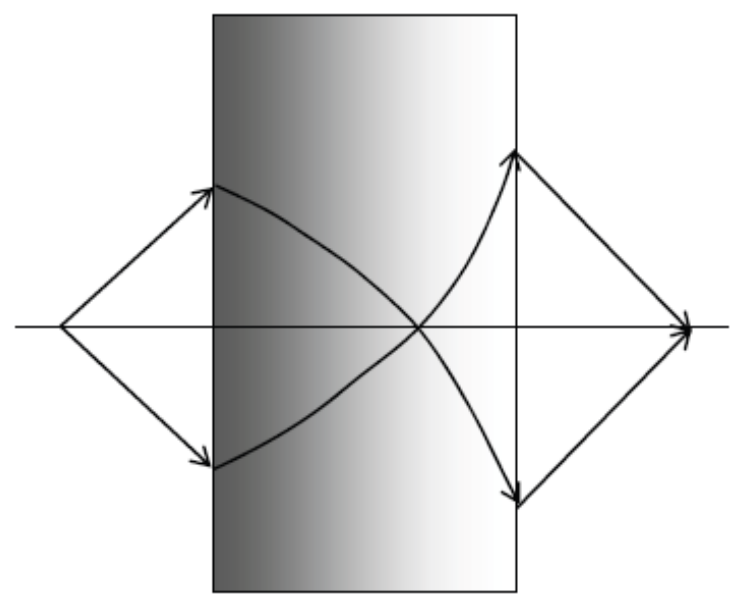

Figure 3. The diagrammatic sketch of a slab with linear negative indices in the second case. Deeper color means greater absolute value of the negative index 


\subsection{Slab with Parabolic Negative Index}

Consider a parabolic negative index slab as

$$
\begin{aligned}
n(z) & =A\left(z-\frac{D}{2}\right)^{2}-n_{0} \\
& =\frac{4\left(n_{0}-1\right)}{D^{2}}\left(z-\frac{D}{2}\right)^{2}-n_{0}
\end{aligned}
$$

where no is a constant (not the refraction index of air), $D$ is the thickness of the slab, and $A=\frac{4\left(n_{0}-1\right)}{D^{2}}$ (see Figure 4; we assume $n_{0}=2, D=2$ as an example). The negative index medium also bends the light to focus twice (see Figure 5). Because of the symmetry of the parabola, light is focused in the center of the slab the first time. The distance of the second focus away from the slab is

$$
\begin{aligned}
& f=\int_{0}^{D} \frac{\cos \theta d z}{\sqrt{n^{2}-\sin ^{2} \theta}}-L \\
& =\int_{0}^{D} \frac{\cos \theta d z}{\sqrt{\left[A\left(z-\frac{D}{2}\right)^{2}-n_{0}\right]^{2}-\sin ^{2} \theta}}-L \\
& =\frac{\left[2 i \cos \theta \sqrt{16-\left(\frac{A D^{2}}{\sin \theta+n_{0}}\right)^{2}}\right.}{\times(i \arcsin \mathrm{h})\left(\frac{D}{2} \sqrt{\left.\frac{A}{\sin \theta-n_{0}} \mid \frac{2 n_{0}}{\sin \theta-n_{0}}-1\right)}\right]}-L
\end{aligned}
$$

where $(a \mid b)$ is an elliptic integral of the first kind. Equation (9) is the analytical result for a parabolic negative index slab. Equation (9) is a restriction of the distance between the optical source and the second focus. The numerical results will be discussed in section 3 .

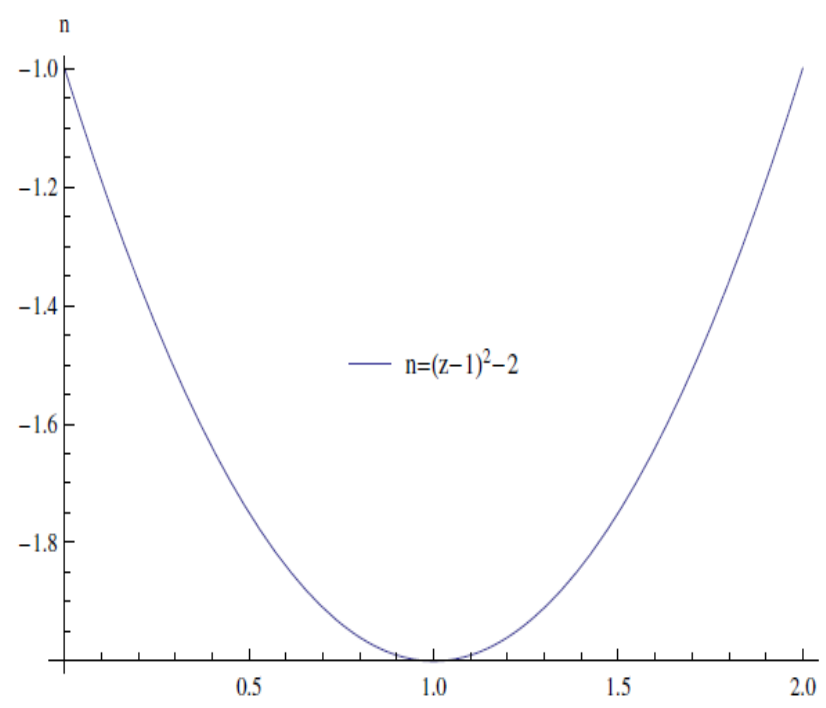

Figure 4. An example of parabolic negative indices $n_{0}=2, D=2$

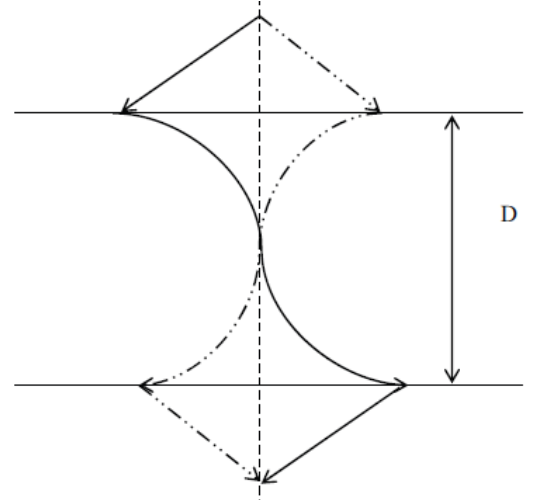

Figure 5. Light is transmitted through a slab with a parabolic negative index. The solid line and dashed line denote the two paths

\section{Numerical Simulation}

For simplicity, we discuss three typical instances of equation (8) $D=6, D=4, D=2\left(n_{0}=2\right)$, and ignore the dimensions here. Figure 6 shows the numerical simulation of these three kinds of slabs with parabolic negative indices. The horizontal coordinates are incident angles, and the longitudinal coordinates represent the length $f+L$. Here, we assume the optical source approaches the slab, $L \ll D$. In Figure 6 , the three colored lines correspond to the three cases $D=6, D=4, D=2$ from top to bottom, respectively. As the incident angle increases, $f+L$ decreases. The numerical simulation curve approximates a parabola oriented downward. According to these curves, to obtain more reasonable and obvious results, $f+L$ should be restricted to the range $0<\theta \leq \frac{\pi}{4}$.

For example, when $D=4, n_{0}=2$, and $\theta=10^{\circ}$, equation (9) gives a restriction $f+L=2.5$. As we assumed $L \ll D$, if $L=1$, we have $f=1.5$. As the optical source approaches the slab, the second focus moves away from the slab, and vice versa. $\theta=0$ are the most obvious cases. In these cases, we notice that the ratio of $D /(f+L)$ are almost fixed. The curves are much steeper for bigger $D$.

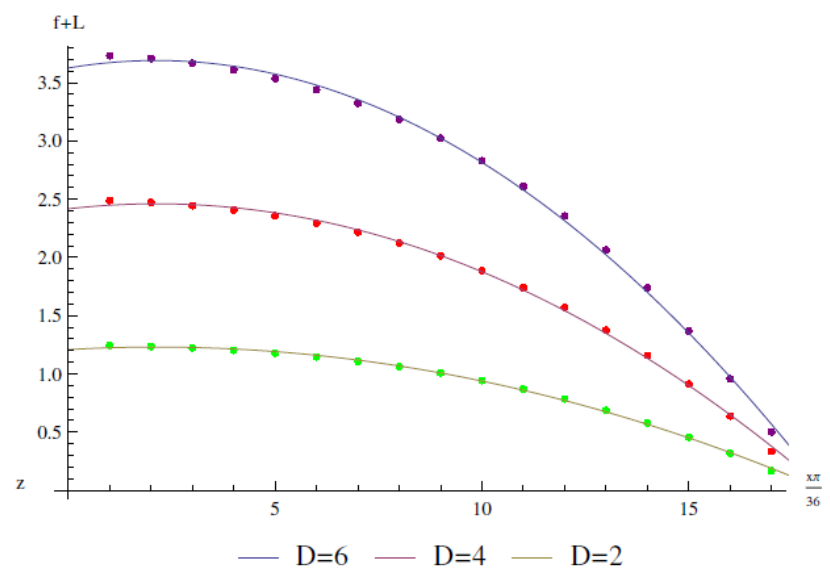

Figure 6. The numerical simulation of a slab with parabolic negative indices $f+L$ vs incident angles. Three colored lines correspond to three cases $D=6, D=4, D=2$ (from top to bottom), respectively. 


\section{Light Interference}

A negative refraction slab with an index of -1 is significant. It can focus lights on one point, thus make a perfect lens. Interference is a common issue with every optical object, including a negative refraction lens. As shown in Figure 7, we suppose the thickness of the negative refraction lens is $d$. The distance between the optical source and lens is $d_{1}$. The negative index of the lens is -1 . When we place the lens in the air, the refraction index of air is 1 . The angles of incidence are $\theta_{1}$ and $\theta_{2}$. The total optical path difference is

$$
\Delta L=\left(\frac{1}{\cos \theta_{2}}-\frac{1}{\cos \theta_{1}}\right)\left(d_{1}-d+d-d_{1}\right)=0,
$$

and the two light beams enhance each other; thus, interference is avoided. The image made by the negative refraction lens with index -1 is the clearest compared with the other negative index lenses. In reality, the absolute value of the negative index is very close to one, and the thickness of negative refraction lens is very small. Therefore, for a negative refraction lens, the total optical path difference can be ignored.

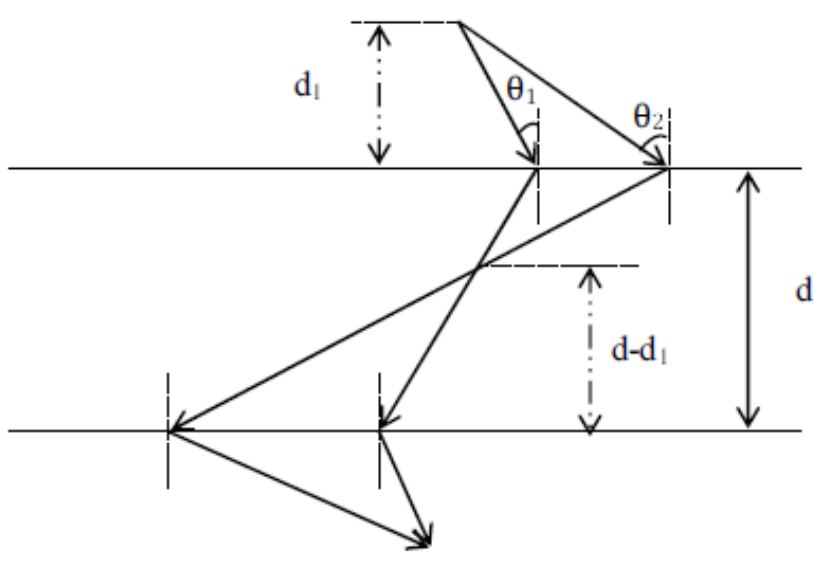

Figure 7. The light interference transmitted through a negative index material. The negative index of the material is -1 in this figure

\section{Conclusion and Discussion}

Because of their superior optical properties and great development potential, negative index materials have become popular and attractive in physics in recent years. However, only negative index materials with a constant index have been discussed. We discuss the inhomogeneity of a NIM-negative refraction slab with a gradient index, and point out that the position of focus changes depending on the incident angle. We conclude negative refraction slab with varying index is not a perfect lens. Only negative refraction slab with single index -1 could focus lights on one point and make a perfect lens. Our research consists of three parts: a negative refraction slab with two different negative indices, and a slab with a linear negative index and one with a parabolic negative index. Through a negative refraction slab, the light from a point source is focused twice. The analytical derivation and numerical simulation demonstrate the limit of the incident angle and explain the relationship between the source position and the second focus. The discussion of a slab with a linear negative index is divided into that concerning an increased and decreased index. The decreased index slab focuses light twice, while the increased index slab only focuses once. The result is optimal when the incident angle of a slab with a parabolic negative index is $0<\theta \leq \frac{\pi}{4}$. Finally, it was determined that light interference through a negative refraction slab could be ignored, in particular for those with a negative index of -1 . In future work, to obtain better optical effects, more types of negative indices should be discussed. In our realization, it will need to require that the geometry of the composite changes on a scale that is much larger than the size of the unit cell yet much smaller than the wavelength. Technique remains a difficulty, further discussions are still needed to realize proposed device.

\section{Acknowledgements}

The research was supported by The Opening Project of Shanghai Key Laboratory of Crime Scene Evidence No.2016XCWZK11.

\section{References}

[1] Veselago, V. G., "The Electrodynamics of Substances with Simultaneously Negative Values of $\varepsilon$ and $\mu$," Sov. Phys. Uspekhi, 10. 509-514. 1968.

[2] Pendry, J. B., "Negative Refraction Makes a Perfect Lens," Phys. Rev. Lett., 85. 3966-3969. 2000.

[3] Pendry, J. B., Holden, A. J., Robbins, D. J., Stewart, W. J., "Magnetism from Conductors and Enhanced Nonlinear Phenomena," IEEE Trans. Microwave Theory Tech., 47. 2075-2084. 1999.

[4] Pendry, J. B., Holden, A. J., Stewart, W. J., Youngs, I., "Extremely Low Frequency Plasmons in Metallic Mesostructures," Phys. Rev. Lett., 76. 4773-4776. 1996.

[5] Pendry, J. B., Holden, A. J., Robbins, D. J., Stewart, W. J., "Low Frequency Plasmons in Thin-Wire Structures," J. Phys.: Condens. Matter, 10. 4785-4809. 1998.

[6] Shelby, R. A., Smith, D. R., Schultz, S., "Experimental Verification of a Negative Index of Refraction," Science, 292. 77-79. 2001.

[7] Cubukcu, E., Zhang, S., Park,Y.-S., Bartal, G., Zhang, X., "Split Ring Resonator Sensors for Infrared Detection of Single Molecular Monolayers," Appl. Phys. Lett., 95. 043113-043113. 2009.

[8] Clark, A. W., Glidle, A., Cumming, D. R. S., Cooper, J. M., "Plasmonic Split-Ring Resonators as Dichroic Nanophotonic DNA Biosensors," J. Am. Chem. Soc., 131. 17615-17619. 2009.

[9] Pryce, I. M., Kelaita, Y. A., Aydin, K., Briggs, R. M., Atwater, H. A., "Compliant Metamaterials for Resonantly Enhanced Infrared Absorption Spectroscopy and Refractive Index Sensing", ACS Nano, 5. 8167-8174. 2011.

[10] Pendry, J. B., Schurig, D., Smith, D. R., "Controlling Electromagnetic Fields", Science, 312. 1780-1782. 2006.

[11] Luo, Y., Zhao, R. K., Fernandez-Dominguez, A. I., Maier, S. A., Pendry, J. B., ? Harvesting light with transformation optics?, Sci. China Inf. Sci., 56. 120401. 2013.

[12] Shen, Y., Chen, Y. Z., "Permittivity and permeability of pentagon configuration molecules with different symmetry breaking and their applications", Sci. China-Phys. Mech. Astron., 60. 070312. 2017.

[13] Zhao, R., Luo, Y., Pendry, J. B., "Transformation optics applied to van der Waals interactions", Sci. Bull., 61. 59. 2016. 
[14] Schurig, D., Mock, J. J., Justice, B. J., Cummer, S. A., Pendry, J. B., Starr, A. F., Smith, D. R., "Metamaterial Electromagnetic Cloak at Microwave Frequencies", Science, 314. 977-980. 2006.

[15] Huang, Y., Gao, L., "Equivalent Permittivity and Permeability and Multiple FanoResonances for Nonlocal Metallic Nanowires", $J$. Phys. Chem. C, 117. 19203-19211. 2013.

[16] Droulias, S., Yannopapas, V., "Broad-Band Giant Circular Dichroism in Metamaterials of Twisted Chains of Metallic Nanoparticles", J. Phys. Chem. C, 117. 1130-1135. 2013.

[17] Ropp, C., Cummins, Z., Probst, R., Qin, S. J., Fourkas, J. T., Shapiro, B., Waks, E., "Positioning and Immobilization of Individual Quantum Dots with Nanoscale Precision", Nano Lett. 10. 4673-4679. 2010.

[18] Smith, D. R., Pendry, J. B., Wiltshire, M. C. K., "Metamaterials and Negative Refractive Index", Science, 305. 788-792. 2004.

[19] Decker, M., Linden, S., Wegener, M., "Coupling Effects in LowSymmetry Planar Split-Ring Resonator Arrays", Opt. Lett., 34. 1579-1581.2009.

[20] I. M. Pryce, K. Aydin, Y. A. Kelaita, R. M. Briggs, H. A. Atwater, Nano Lett. 10 (2010) 4222-4227.

[21] W. T. Chen, P. C. Wu, C. J. Chen, C. T. Hsiao, K. Y. Yang, S. Sun, L. Zhou, G. Y. Guo, N. I. Zheludev, D. P. Tsai, SPIE Newsroom, 2011.

[22] Liu, N., Liu, H., Zhu, S., Giessen, H., "Stereometamaterials", Nat. Photonics, 3.157-162. 2009.
[23] Fleming, G. R., Wolynes, P. G., "Chemical Dynamics in Solution", Phys. Today, 43. 36-43. 1990.

[24] A. Szabo, N. S. Ostlund, Modern Quantum Chemistry, Introduction to Advanced Electronic Structure Theory, Dover: New York, 1996.

[25] H. H. Greenwood, Computing Methods in Quantum Organic Chemistry, Wiley-Interscience: Weinheim, Germany, 1972.

[26] Pantazis, D. A., McGrady, J. E., "A Three-State Model for the Polymorphism in Linear Tricobalt Compounds", J. Am. Chem. Soc 128. 4128-4135. 2006.

[27] Pyrka, G. J., El-Mekki, M., Pinkerton, A. A., "Structure of the Linear Trinuclear Copper Complex, Dichlorotetrakis-(di-2pyridylamido)tricopper", J. Chem. Soc., Chem. Commun., 84-85. 1991.

[28] Peng, S.-M., Wang, C.-C., Jang, Y.-L., Chen, Y.-H., Li, F.-Y., Mou, C.-Y., Leung, M.-K. J., "One-Dimensional Metal String Complexes", Magn. Magn. Mater, 209. 80-83. 2000.

[29] Tsai, T. -W., Huang, Q. -R., Peng, S. -M., Jin, B. -Y., "Smallest Electrical Wire Based on Extended Metal-Atom Chains", J. Phys. Chem. C, 114. 3641-3644. 2010.

[30] Shen, Y., Ai, Q., "Optical properties of drug metabolites in latent fingermarks", Sci. Rep., 6. 20336. 2016. 